IOS Press

\title{
Time for a New 3-D Image for Globus Pallidus Internus Deep Brain Stimulation Targeting and Programming
}

\author{
Joshua K. Wong ${ }^{\mathrm{a}, *}$, Justin D. Hilliard ${ }^{\mathrm{b}}$, Vanessa M. Holanda ${ }^{\mathrm{c}, \mathrm{d}}$, Aysegul Gunduz ${ }^{\mathrm{a}, \mathrm{e}}$, \\ Aparna Wagle Shukla ${ }^{\mathrm{a}}$, Kelly D. Foote ${ }^{\mathrm{b}}$ and Michael S. Okun ${ }^{\mathrm{a}}$ \\ ${ }^{\text {a }}$ Fixel Institute for Neurological Diseases, Department of Neurology, University of Florida, Gainesville, FL, \\ USA \\ ${ }^{\mathrm{b}}$ Fixel Institute for Neurological Diseases, Department of Neurosurgery, University of Florida, Gainesville, FL, \\ USA \\ ${ }^{\mathrm{c} C e n t e r ~ o f ~ N e u r o l o g y ~ a n d ~ N e u r o s u r g e r y ~ A s s o c i a t e s ~(C E N N A), ~ B P ~-~ A ~ B e n e f i c e ̂ n c i a ~ P o r t u g u e s a ~ d e ~ S a ̃ o ~ P a u l o, ~}$ \\ São Paulo, SP, Brazil \\ ${ }^{\mathrm{d}}$ Department of Neurosurgery, Mayo Clinic, Jacksonville, FL, USA \\ ${ }^{\mathrm{e}}$ Department of Biomedical Engineering, University of Florida, Gainesville, FL, USA
}

Accepted 2 August 2021

Pre-press 20 August 2021

\begin{abstract}
Deep brain stimulation (DBS) is an effective neuromodulatory therapy for Parkinson's disease (PD). Early studies using globus pallidus internus (GPi) DBS for PD profiled the nucleus as having two functional zones. This concept disseminated throughout the neuromodulation community as the "GPi triangle". Although our understanding of the pallidum has greatly evolved over the past 20 years, we continue to reference the triangle in our clinical decision-making process. We propose a new direction, termed the spatial boundary hypothesis, to build upon the 2-dimensional outlook on GPi DBS. We believe an updated 3-D GPi model can produce more consistent, positive patient outcomes.
\end{abstract}

Keywords: Deep brain stimulation, DBS, globus pallidus, GPi, Parkinson's disease, targeting, neuromodulation

\section{INTRODUCTION}

Deep brain stimulation (DBS) is an effective neuromodulatory therapy for select movement disorders such as Parkinson's disease (PD), essential tremor and dystonia [1-3]. The globus pallidus internus (GPi) is one of the most frequently used targets

\footnotetext{
${ }^{*}$ Correspondence to: Joshua K. Wong, MD, 3009 Williston Road, Gainesville, FL 32608, USA. Tel.: +1 352294 5400; E-mail: joshua.wong@neurology.ufl.edu.
}

for PD DBS. There is, however, a significant and surprising amount of variability in neurosurgical techniques, trajectory planning, and intraoperative localization of the GPi DBS target. Advanced imaging techniques, computational models, probabilistic stimulation atlases, and connectivity mapping have collectively expanded our understanding of the GPi target and have transformed a 20 -year-old concept from 2-D to 3-D. We believe that application of an updated 3-D GPi targeting model can provide a better understanding of GPi DBS therapy and produce more consistent, positive patient outcomes. 


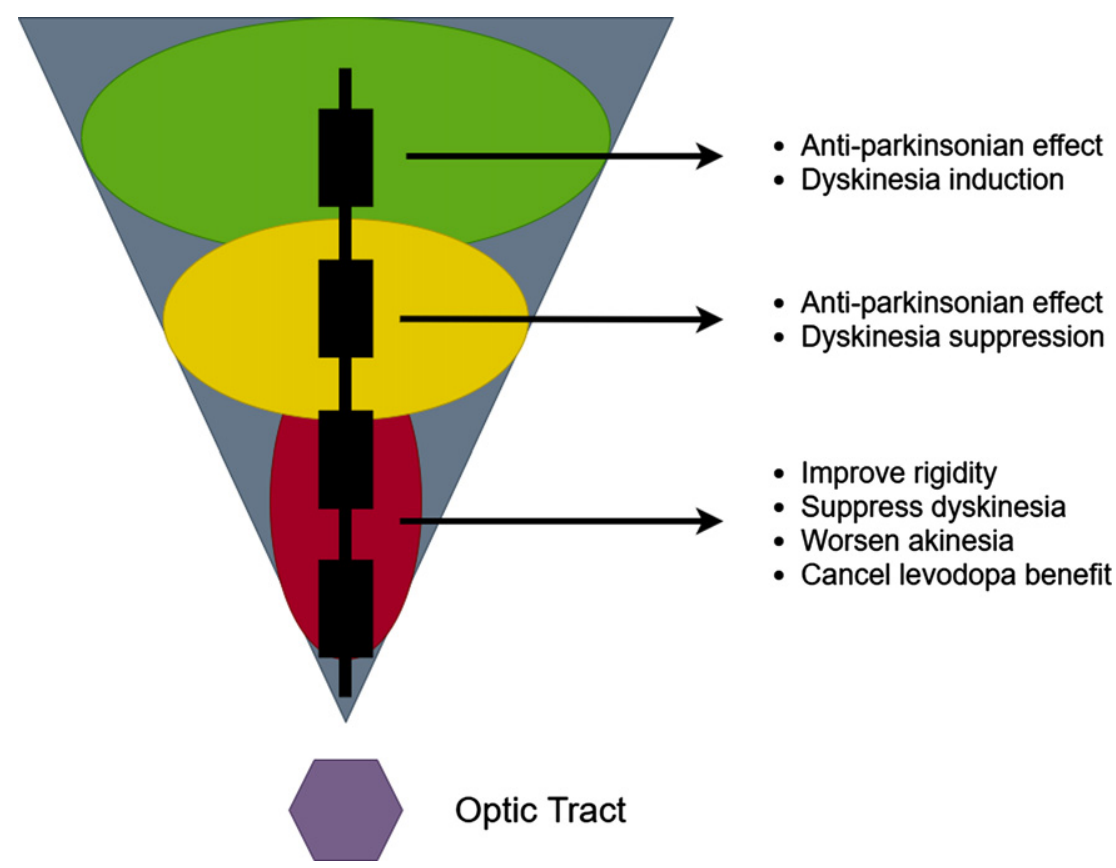

Fig. 1. The 2-D two zone GPi DBS image.

\section{A BRIEF HISTORY}

Prior to the advent of PD GPi DBS in 1994, the standard option for neurosurgical intervention was ablative or lesional therapy [4, 5]. Lesions of the GPi (pallidotomies) provided effective relief for the classical parkinsonism symptoms, improvements in motor fluctuations and suppression of levodopa induced dyskinesia [5, 6]. However, as pallidotomies became increasingly performed, it was appreciated that bilateral lesional procedures were associated with unacceptable risks of pseudobulbar symptoms and other adverse effects [7, 8]. Following the pallidotomy era, bilateral GPi DBS emerged as a safer alternative to the bilateral lesional approach [9].

\section{THE CLASSICAL TWO-ZONE 2-D GPi IMAGE}

In an effort to optimize outcomes of GPi DBS, experts have endeavored to spatially characterize the effects of pallidal stimulation. Krack and Bejjani simultaneously described a largely 2-D representation of the GPi; their description was of two functional zones $[10,11]$. This characterization led to the widespread use of the classical 2-D "GPi triangle" (Fig. 1). This figure has been widely circulated but has many limitations [12]. The triangle is characterized by a dorsal GPi zone which was considered anti-parkinsonian, however the authors conceded that dorsal zone stimulation could also induce dyskinesia. Stimulation in the ventral GPi zone has been associated with improvements in rigidity and dyskinesia, but also with worsening of akinesia. Furthermore, stimulation in the ventral zone was said to "cancel" the levodopa benefit. A 1-D GPi "sweet spot" between the dorsal and ventral zones was proposed as the best target.

The 20 years following the 2-D descriptions of GPi DBS have added critical new insights. Electrophysiology, structural MRI, functional MRI, and computation modeling have collectively demonstrated that the previous 2-D mindset, and the twozone hypothesis was too simplistic and would in some cases contribute to suboptimal placement of DBS leads. We propose as a modern alternative, the spatial boundary hypothesis for GPi DBS (Fig. 2).

\section{SPATIAL CONSIDERATIONS FOR GPi TARGETING}

The anatomy of the GPi is inadequately described with a 1-D or 2-D representation. The internal capsule (IC) is a well-defined white matter pathway 


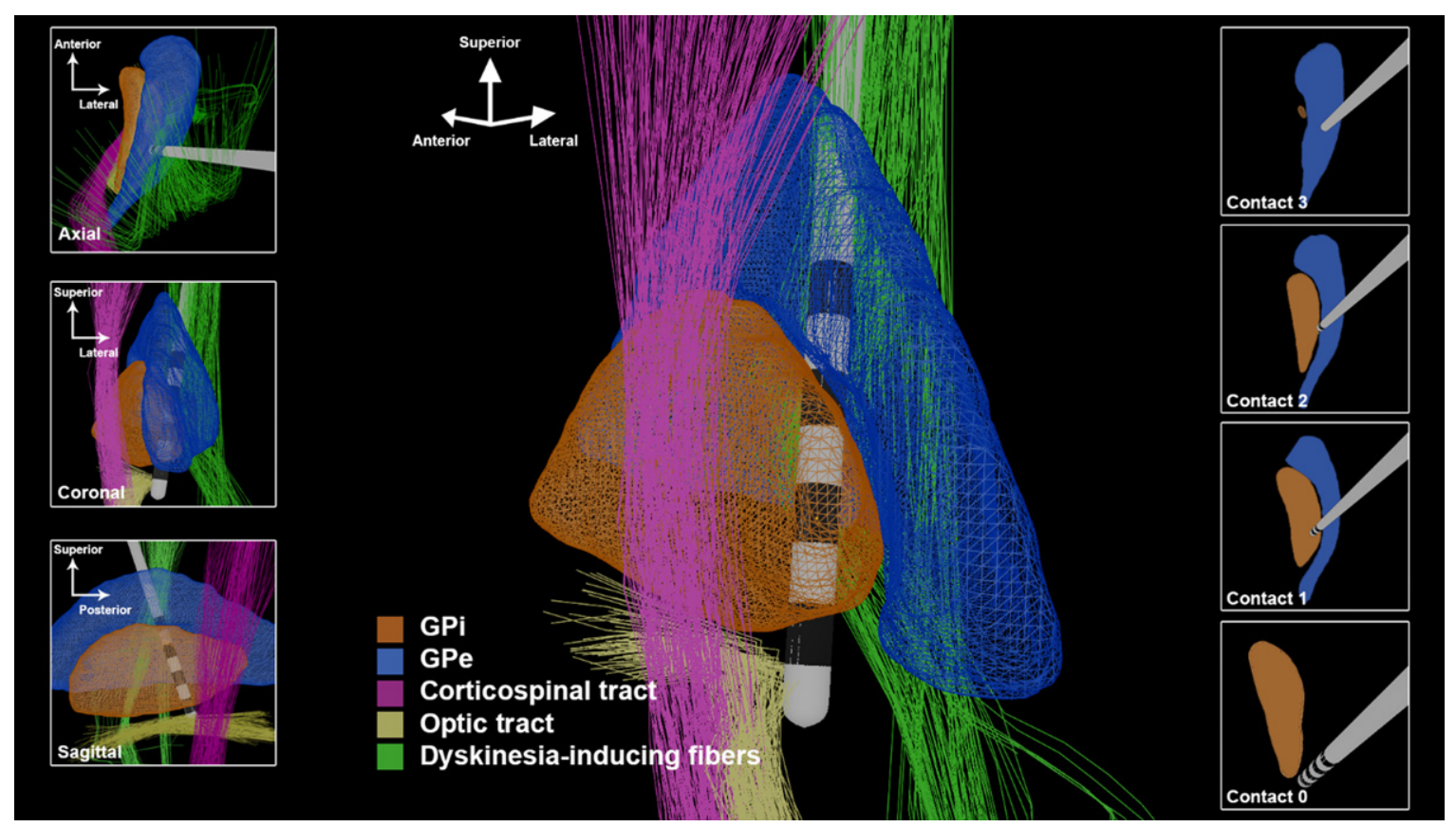

Fig. 2. The 3-D GPi DBS image utilizing trajectories and spatial boundaries for targeting and programming. The lead depicted in this figure has $1.5 \mathrm{~mm}$ spacing between contacts and a contact height of $1.5 \mathrm{~mm}$.

traversing the medial and posterior borders of the GPi. Side effects from IC stimulation range broadly from tonic muscle contractions to dysarthria and tend to be the most significant dose-limiting adverse effects of GPi stimulation [13]. Thus, the trajectory of a GPi DBS lead in both the anterior-posterior and medial-lateral planes will determine the proximity of the DBS contacts to the IC. This establishes the thresholds for stimulation induced adverse effects and the "programmability" of the implanted lead, which is a significant determinant of DBS outcome and patient satisfaction. In our experience, postoperative quality assurance through image-based lead localization and clinical thresholds for capsular side effects can provide helpful feedback for trajectory planning. Building upon pallidotomy techniques that suggest a $3-4 \mathrm{~mm}$ separation from IC evoked motor responses, we found a distance of approximately $3 \mathrm{~mm}$ between the DBS contacts and the IC provides an optimal side effect threshold [14].

GPi DBS is widely known to effectively suppress problematic dyskinesias, and has been advocated as a potentially preferable option to STN DBS for patients with severe dyskinesias [4]. However, GPi DBS can also induce dyskinesias, as initially described by Krack and Bejjani [10, 11]. The observation of this phenomenon has been confirmed through additional reports and observations [15]. Recently, a connectivity analysis demonstrated that dorsal stimulation near the GPi and globus pallidus externus (GPe) border was strongly associated with the occurrence of stimulation induced dyskinesias (Fig. 2, "Dyskinesiainducing fibers") [16]. In this study, Tsuboi et al. reported that $29 \%$ of participants with GPi DBS experienced stimulation induced dyskinesias. To be clear, stimulation in this region is commonly therapeutically beneficial, but may induce dyskinesia in a minority of patients. In the subset of patients in whom dyskinesias are induced with dorsolateral stimulation, stimulation at a more ventromedial contact can typically suppress dyskinesia.

The optic tract, positioned ventromedially to the $\mathrm{GPi}$, is another useful anatomic landmark for GPi DBS targeting. The optic tract is readily identifiable on MR imaging, and in our experience, selection of a target laterally adjacent to the optic tract produces optimal results. Intraoperatively, visual evoked potentials (VEPs) can provide information about the chosen trajectory [13]. The appreciation of "louder" intra-operative VEPs usually suggests a more medial and/or ventral location. Similarly, the amount of current that must be delivered to elicit the visual phenomenon of phosphenes can be used to determine 
the mediolateral and ventral position of the deepest DBS contact intraoperatively. If the threshold for phosphene production is too low, the lead may be excessively medial If the phosphene threshold is too high, or phosphenes are not elicited, the lead may be excessively lateral or superficial. The pallidotomy literature proposed a threshold of greater than $2 \mathrm{~V}$ for trajectories directly targeting the optic tract [17]. However, with a lateral targeting approach, we found a threshold of 1-3 mA to be the optimal range.

Stimulation of the posteroventral GPi has been associated with improvement in both rigidity and levodopa-induced dyskinesia. However, posteroventral GPi DBS has been observed in some cases to worsen akinesia, worsen gait and to reduce the anti-parkinsonism benefit derived from levodopa replacement therapy. Posterodorsal stimulation, in contrast, is associated with improved akinesia, rigidity and gait, but has been associated with the generation of stimulation induced dyskinesia. Using 3-D imaging techniques, Middlebrooks et al. applied structural connectivity-based segmentation of the GPi [18]. They observed that a more ventral area of stimulation was indeed associated with a greater reduction in parkinsonism, but that this effect was more a function of the connectivity of the stimulated region rather than stimulation in the dorsal-ventral plane.

A comprehensive examination of the 3-D representation of GPi and surrounding structures has revealed the critical importance of the anterior-posterior and lateral-medial angles chosen by the neurosurgeon for optimizing the DBS lead trajectory. The available trajectory angles can be constrained by avoidance of superficial cortical veins and the ventricles. The degree of lateral-medial angulation will influence the spatial distribution of the DBS contacts and their proximity to the IC or to the GPi-GPe border region. A more vertical lateral to medial trajectory is desirable in GPi targeting because it is more coaxial with the target and allows for more DBS contacts to be positioned within the posterolateral GPi. If the lateral-medial entry angle exceeds 5 degrees due to superficial anatomic constraints, a medial adjustment should be considered in order to keep the center of the electrode array within the GPi. If the DBS target is selected solely based on the location of the ventral contact and the lateralmedial trajectory angle is increased, failure to make a corrective medial target adjustment will result in suboptimal lateral positioning of the dorsal DBS contacts.

\section{THE MODERN APPROACH TO GPi DBS: A NEW IMAGE EMERGES}

We argue that it is time to transition from 1-D and 2D GPi images to 3-D illustrations that provide essential information to support both DBS targeting and programming. In the anterior-posterior plane, a DBS lead positioned too anteriorly may have reduced therapeutic benefit or result in a need for increased current delivery, resulting in more rapid battery depletion. A lead positioned too posteriorly will spread current into the internal capsule at low thresholds, resulting in stimulation induced capsular side effects that can limit therapeutic stimulation and benefit. Mild capsular side effects may also emerge and mimic worsening of parkinsonism, which may explain the previously described "cancelling of levodopa" effect reported in the original GPi DBS publications. A GPi lead that is excessively medial might also produce dose-limiting capsular side effects. On the other hand, a lead that is too far lateral typically results in a reduction of benefit or necessitates higher energy requirements to deliver therapeutic stimulation into the GPi region. A laterally positioned DBS lead is also more likely to produce stimulation induced dyskinesias, especially when activating the more dorsal contacts that end up in the GPe. In the dorsal-ventral plane, more dorsal stimulation can result in stimulation induced dyskinesia, while more ventral stimulation can result in capsular or visual side effects.

The ability to map the 3-D architecture of the GPi region has vastly improved over the last two plus decades. Meticulous attention to these preoperative targeting considerations (and appropriate targeting adjustments when intra-operative physiologic testing is performed) can expand the options for post-operative programming and management, improving therapeutic benefit and patient satisfaction. Though 1-D and 2-D representations of GPi DBS previously shaped the field, it is time to graduate to a 3-D GPi representation. Future GPi DBS targeting and programming should focus on a more careful selection of the lead trajectory and on a deliberate attempt to stimulate or to avoid specific fiber bundles. This approach has the potential to optimize global outcomes of GPi DBS and minimize adverse effects.

\section{CONFLICT OF INTEREST}

There are no conflicts of interest to this manuscript. 


\section{REFERENCES}

[1] Deep-Brain Stimulation for Parkinson's Disease Study Group, Obeso JA, Olanow CW, Rodriguez-Oroz MC, Krack P, Kumar R, Lang AE (2001) Deep-brain stimulation of the subthalamic nucleus or the pars interna of the globus pallidus in Parkinson's disease. N Engl J Med 345, 956-963.

[2] Benabid AL, Pollak P, Gervason C, Hoffmann D, Gao DM, Hommel M, Perret JE, de Rougemont J (1991) Long-term suppression of tremor by chronic stimulation of the ventral intermediate thalamic nucleus. Lancet 337, 403-406.

[3] Vidailhet M, Vercueil L, Houeto J-L, Krystkowiak P, Benabid A-L, Cornu P, Lagrange C, Tézenas du Montcel S, Dormont D, Grand S, Blond S, Detante O, Pillon B, Ardouin C, Agid Y, Destée A, Pollak P (2005) Bilateral deep-brain stimulation of the globus pallidus in primary generalized dystonia. N Engl J Med 352, 459-467.

[4] Siegfried J, Lippitz B (1994) Bilateral chronic electrostimulation of ventroposterolateral pallidum: A new therapeutic approach for alleviating all parkinsonian symptoms. Neurosurgery 35, 1126-1129; discussion 1129-1130.

[5] Svennilson E, Torvik A, Lowe R, Leksell L (1960) Treatment of parkinsonism by stereotatic thermolesions in the pallidal region. A clinical evaluation of 81 cases. Acta Psychiatr Scand 35, 358-377.

[6] Laitinen LV, Bergenheim AT, Hariz MI (1992) Leksell's posteroventral pallidotomy in the treatment of Parkinson's disease. J Neurosurg 76, 53-61.

[7] Scott R, Gregory R, Hines N, Carroll C, Hyman N, Papanasstasiou V, Leather C, Rowe J, Silburn P, Aziz T (1998) Neuropsychological, neurological and functional outcome following pallidotomy for Parkinson's disease. A consecutive series of eight simultaneous bilateral and twelve unilateral procedures. Brain 121 (Pt 4), 659-675.

[8] Ghika J, Ghika-Schmid F, Fankhauser H, Assal G, Vingerhoets F, Albanese A, Bogousslavsky J, Favre J (1999) Bilateral contemporaneous posteroventral pallidotomy for the treatment of Parkinson's disease: Neuropsychological and neurological side effects. Report of four cases and review of the literature. J Neurosurg 91, 313-321.

[9] Ghika J, Villemure J-G, Fankhauser H, Favre J, Assal G, Ghika-Schmid F (1998) Efficiency and safety of bilateral contemporaneous pallidal stimulation (deep brain stimulation) in levodopa-responsive patients with Parkinson's disease with severe motor fluctuations: A 2-year follow-up review. J Neurosurg 89, 713-718.

[10] Krack P, Pollak P, Limousin P, Hoffmann D, Benazzouz A, Le Bas JF, Koudsie A, Benabid AL (1998) Opposite motor effects of pallidal stimulation in Parkinson's disease. Ann Neurol 43, 180-192.

[11] Bejjani B, Damier P, Arnulf I, Bonnet AM, Vidailhet M, Dormont D, Pidoux B, Cornu P, Marsault C, Agid Y (1997) Pallidal stimulation for Parkinson's disease: Two targets? Neurology 49, 1564-1569.

[12] Marks WJ Jr (2010) Deep Brain Stimulation Management, Cambridge University Press, Cambridge.

[13] Au KLK, Wong JK, Tsuboi T, Eisinger RS, Moore K, Lemos Melo Lobo Jofili Lopes J, Holland MT, Holanda VM, PengChen Z, Patterson A, Foote KD, Ramirez-Zamora A, Okun MS, Almeida L (2021) Globus pallidus internus (GPi) deep brain stimulation for Parkinson's disease: Expert review and commentary. Neurol Ther 10, 7-30.

[14] Lozano A, Hutchison W, Kiss Z, Tasker R, Davis K, Dostrovsky J (1996) Methods for microelectrode-guided posteroventral pallidotomy. J Neurosurg 84, 194-202.

[15] Elkouzi A, Tsuboi T, Burns MR, Eisinger RS, Patel A, Deeb W (2019) Dorsal GPi/GPe stimulation induced dyskinesia in a patient with Parkinson's disease. Tremor Other Hyperkinet Mov (N Y) 9, doi: 10.7916/tohm.v0.685

[16] Tsuboi T, Charbel M, Peterside DT, Rana M, Elkouzi A, Deeb W, Ramirez-Zamora A, Lemos Melo Lobo Jofili Lopes J, Almeida L, Zeilman PR, Eisinger RS, Foote KD, Okromelidze L, Grewal SS, Okun MS, Middlebrooks EH (2021) Pallidal connectivity profiling of stimulationinduced dyskinesia in Parkinson's disease. Mov Disord 36, 380-388.

[17] Bonaroti EA, Rose RD, Kondziolka D, Baser S, Lunsford LD (1997) Flash visual evoked potential monitoring of optic tract function during macroelectrode-based pallidotomy. Neurosurg Focus 2, e4.

[18] Middlebrooks EH, Tuna IS, Grewal SS, Almeida L, Heckman MG, Lesser ER, Foote KD, Okun MS, Holanda VM (2018) Segmentation of the globus pallidus internus using probabilistic diffusion tractography for deep brain stimulation targeting in Parkinson disease. AJNR Am J Neuroradiol 39, $1127-1134$. 